\title{
An Automated Environment for Hardware Testing Using PXI Instrumentation and LabVIEW Software
}

\author{
Vladimir D. Ćatić, Natalija M. Lukić, Iva M. Salom, Vukašin P. Ristić, Milenko M. Kabović, \\ Bratislav Ž. Planić, and Nikola M. Nenadić
}

\begin{abstract}
Reliable and fast testing was a requirement in the serial production of the advanced wireless microphone MIKME. Hence, a system for automatic testing, fully adapted for the product, was developed. The system comprises: 1) a specially designed test fixture - bed-of-nails, on which the device under test is placed, 2) the PXI platform and 3) an application developed in LabVIEW environment. Throughout the design of this system the goal was to achieve maximal automation of the entire test procedure in order to minimize the influence of human factor, and therefore save time and significantly reduce the possibility of error.
\end{abstract}

Keywords - automated hardware testing, LabVIEW, PXI, test procedures, virtual instrumentation.

\section{INTRODUCTION}

$\mathrm{T}$ EST strategy is critical for reducing the cost and maximizing the efficiency of complex products development and manufacturing. Therefore automatic test equipment (ATE) has an immense usage in the electronic industry.

Paper received May 8, 2017; revised August 11, 2017; accepted August 21, 2017. Date of publication December 25, 2017. The associate editor coordinating the review of this manuscript and approving it for publication was Prof. Vujo Drndarević.

This paper is a revised and expanded version of the paper presented at the 24th Telecommunications Forum TELFOR 2016 [12].

This work received a partial support from the Serbian Ministry of Education, Science and Technological Development, projects TR32038 and III44003.

Vladimir D. Ćatić, is with the Mihailo Pupin Institute, Volgina 15, 11060 Belgrade, Serbia; (phone: 381-11-2773545, e-mail: vladimir.catic@pupin.rs).

Natalija M. Lukić is with the Mihailo Pupin Institute, Volgina 15, 11060 Belgrade, Serbia; (phone: 381-11-677-27-55, e-mail: natalija.lukic@pupin.rs).

Corresponding author, Iva M. Salom is with the Mihailo Pupin Institute, Volgina 15, 11060 Belgrade, Serbia; (phone: 381-11-677-2755, e-mail: iva.salom@pupin.rs).

Vukašin P. Ristić is with the Mihailo Pupin Institute, Volgina 15, 11060 Belgrade, Serbia; (phone: 381-11-677-27-55, e-mail: vukasin.ristic@pupin.rs).

Milenko M. Kabović is with the Mihailo Pupin Institute, Volgina 15, 11060 Belgrade, Serbia; (phone: 381-11-677-27-55, e-mail: milenko.kabovic@pupin.rs).

Bratislav Ž. Planić is with the Mihailo Pupin Institute, Volgina 15, 11060 Belgrade, Serbia; (phone: 381-11-677-27-55, e-mail: bratislav.planic@pupin.rs).

Nikola M. Nenadić is with the Mihailo Pupin Institute, Volgina 15 11060 Belgrade, Serbia; (phone: 381-11-677-27-55, e-mail: nenadic.nikola@gmail.com).
ATE implies a system which is used to test a device using automatic operations to promptly acquire measurements and update the results of the testing. Automatic test systems are widely spread in manufacturing of electronics to test components and systems after production. Various components can be verified, from simple ones (resistors, capacitors, inductors...) to integrated circuits, printed boards and even complex ones, such as assembled electronic systems. ATE systems are designed to reduce the time needed for verification of the proper functioning of a device and to quickly find errors before the device is commercially used. By these means, control of quality is improved in production, decreasing the number of defect devices on the market.

Possible causes of malfunctions can be errors occurred during an assembly process (for example: poor soldering contact or short circuit), faulty components populated etc. Tests usually consist of the basic electrical measurements (galvanic isolation, contact resistance, power supplies, current consumption, clock source frequency...), but also can include more complex tests, such as interface check, protocol verification, memory tests, as well as a complete functional test. Although in some particular cases unit testing can be performed manually, using traditional instruments, such as multimeter, frequency counter, oscilloscope, automatic test procedures are much more efficient and reliable, and less prone to human errors due to negligence and fatigue, thus improving time-to-market and dramatically reducing production cost [1]-[5].

In this paper an automated system for hardware testing in the production lines of the MIKME device is presented. MIKME is an advanced battery powered standalone wireless high-quality audio recording device, which meets several requirements in a single device: it contains a highquality condenser capsule providing studio grade signal quality; it has a built-in internal memory providing recording, playback, record-on-top and AAC (Advanced Audio Coding) encoding functions, as well as sharing files via USB interface; it provides real-time wireless streaming to iOS (Apple mobile operating system) based devices via Bluetooth interface, using a designed application to edit, mix and share recorded tracks [6], [7].

These demanding features imposed a complex hardware architecture that comprises two functional boards, and a flex board connecting them, a large number of components on a relatively small surface and a dense track routing, mix 
of analog and digital signals. Additionally, analog signals have a high dynamic range. Such a hardware structure increases a risk of errors during the production, thus the testing of each part of the system has to be carefully performed. For that reason a complex system for automated testing in production lines, based on the PXI modular instruments [8] and a LabVIEW [9] application, was designed. At the end of a successfully performed test the device firmware is downloaded as well, and thus after the tests verified units are ready for assembling in a final product.

\section{AUTOMATED TEST ENVIRONMENT}

For the purpose of automated hardware unit testing of the MIKME device, the utilized equipment is connected into the test system, whose general structure is presented in Fig. 1. The hardware configuration, shown in detail in Fig. 2, contains a computer - the main controller of the system with a specially designed LabVIEW application that manages testing by driving the PXI instrumentation and communicating to the Units Under Test (MIKME boards) using a specially developed ATE firmware with the communication protocol and test routines. Additionally, the LabVIEW application communicates with a database (DB) on the server, obtaining the basic information from the database and writing the test results to it. A specially designed test fixture, so called bed-of-nails (BoN) [10], [11], presents the interface between the components of the system.

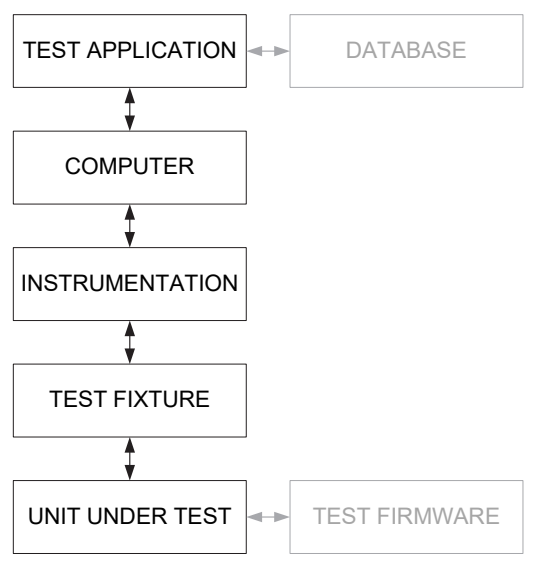

Fig. 1. General block diagram of an automated test environment.

\section{A. Units under test}

Units Under Test (UUT) comprises the two boards of the MIKME device: the top and the main board [7]. Both boards are based on a powerful 32-bit ARM Cortex M4 processor. The top board consists of analog audio components: A/D converter, D/A converter and analog circuits for control of

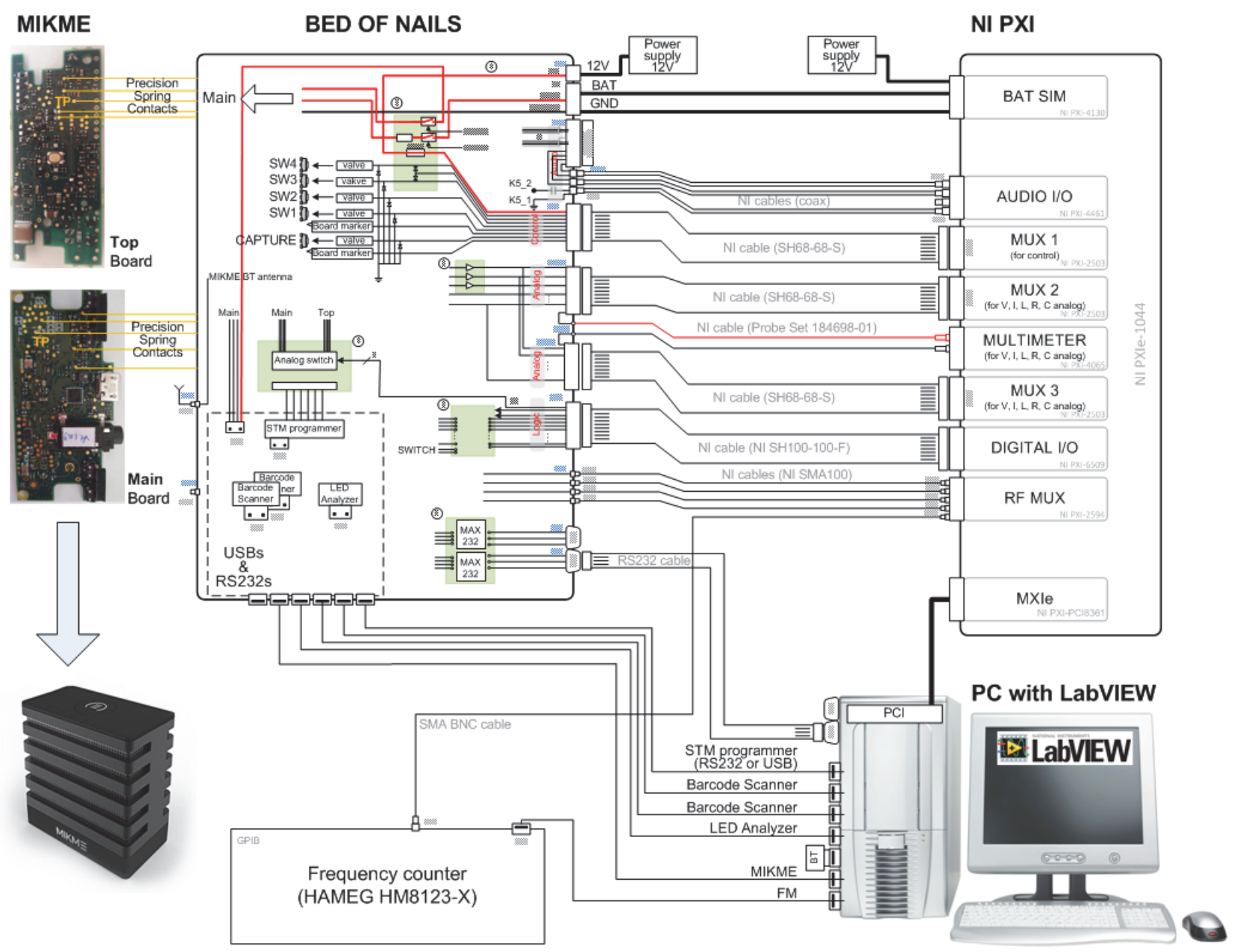

Fig. 2. Schematic representation of the proposed system hardware implementation. 
audio signal gains. The processor performs the processing of the audio signal, low cut filtering, automatic gain control, MP4 audio encoding and decoding, stream synchronization buffers, etc.

The main board is a digital board. It is used to gather digital audio data from the top board, to write the data to the eMMC (Embedded Multi-Media Controller) memory and, in wireless recording use-case, to send the data via a secure Bluetooth connection to an iOS mobile device. The main board can also receive commands and audio data from iOS devices, decode it and playback it on the top board. In addition to the Bluetooth, USB connection can be used both for data transfer to the computer and for the battery charging.

The boards can be tested simultaneously or separately using an already verified top or main board.

On both boards there are test points made of copper covered with a thin and soft alloy film, so designed as to ensure proper connection of the test probes when the unit is placed in the BoN.

\section{B. Bed-of-nails}

A bed-of-nails tester is a traditional electronic test fixture which consists of a large number of spring-loaded test probes [10]. Test probes are placed in the probe plate made of heatproof and robust glass fiber FR4 material and wired to a defined intermediate interface [11]. This wiring was done by wrapping or soldering. Wires are then linked to the appropriate modules or to other external devices, directly or via a specially designed adapter board that provides required interfaces between different signals (the adapter board components are presented in green rectangles in Fig. 2). On the other side of the test probes, test points of the main and the top board are connected.

In addition to the probes and the wirings, there are other instruments set inside the BoN, such as optic fibers (used for analyzing the LEDs) and pressure guidelines with valves, used for switching the actuators that press the buttons on the boards (pushbuttons test).

\section{NI PXI modular instrumentation}

PXI (PCI eXtensions for Instrumentation) is an open platform designed for testing, measurement and control, based on the PCI computer bus. Designed as a modular platform, PXI allows building of a flexible test environment through a wide range of available modular instruments. National Instruments PXI platform is used for the testing, with the following modules [8]:

- NI PXI-PCI8361 (MXI card) - direct PC control of PXI via the PCI slot;

- NI PXI-4130 (Source Measure Unit) - providing voltage supply for the boards and simulating battery (when the board is under USB voltage supply, the card can sink current and behave as a battery); additionally, readings of voltage and current can be made at every given moment of time;

- NI PXI-2503 (Matrix Relay Switch) - 3 pieces, two in combination with DMM (Digital Multimeter Measurement; outputs are connected to DMM) for measurements between different test points and one for the control of the actuators (valves for pushbuttons and board markers; input connected to $12 \mathrm{~V}$ power supply);

- NI PXI-4065 (DMM) - providing measurements of required resistances and voltages;

- NI PXI-6509 (Digital Input/Output) - generating 0 or $5 \mathrm{~V}$ in the case when the pin is set as an output (used for control of relays, FETs, etc.), and when set as an input, it is used for reading an input binary signal;

- NI PXI-2594 (RF Switch) - used for multiplexing RF signals (4 input channels connected to an output);

- NI PXI-4461 (Dynamic Signal Analyzer) simultaneously generating and acquiring signals for verification of audio signal quality.

\section{Other Devices}

In addition to the PXI modules, there are several other instruments used in this system:

- Barcode scanners are fixed on the BoN and their purpose is to read ID numbers from boards placed inside;

- LED analyzer executes acquisition of color and intensity of LEDs using optical guide lines;

- Frequency counter measures the frequencies of both main and top processors (their oscillators indirectly) as well as the Bluetooth frequency;

- Flasher (J-Flash) programs boards with the ATE (after resistance and voltage tests) and the production firmware (at the end of the testing process);

- Bluetooth dongle checks the device visibility and connectivity using the device's MAC address;

- Solenoid valves used in the pushbutton tests;

- Board markers mark boards that passed the testing.

\section{E. ATE firmware}

For the purpose of testing the boards within the ATE, a special firmware has been developed both for the top and for the main board. After resistances and voltages have been tested with a favorable outcome, the firmware is flashed on each board providing a serial (UART) connection to the main board. In order to simplify the communication, the main board receives all the commands for the tests and forwards corresponding messages towards the top board via a serial connection. There is a determined UART protocol for sending and receiving commands. The length of a command is 10 bytes. The first two bytes of the message represent the start frame delimiter (SFD; 0xAA and 0x55). If the byte 0xAA appears in the structure of the message, it is coded with 0xAA 0x00. The next two bytes define the direction of the message (the source address and the destination). The fifth byte represents a label of the given command. The rest of the bytes represent a payload. An example of UART command is shown in Fig. 3.

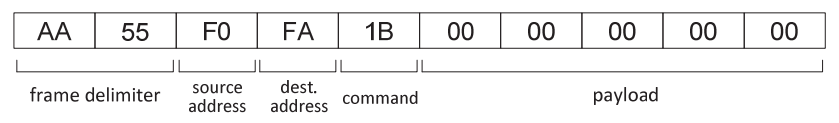

Fig. 3 An example of the UART command (main board blue LED on).

After the command is received the main board responds. The main board first sends the acknowledgement, usually followed by one or two messages consisting of the results or 
a notification that the execution was finished (these results are stored in the payload of the message). The sixth byte of each message is reserved for the checksum. The commands are used to start Bluetooth chip, turn on LEDs, check if the buttons are pressed, check the internal voltage measurements, set audio gains, etc. Additionally, there is a different command for writing bytes to the one-time programmable (OTP) memory of the processor. First, the command for OTP writing is sent, and then the OTP bytes follow. The OTP bytes comprise information about the board, such as serial number, hardware and software version, etc.

\section{F. LabVIEW application}

While PXI modules together with additional instrumentation directly perform acquisition and issue command signals, a developed LabVIEW 2012 [9] application automatically controls the test process and manages the National Instruments virtual instrumentation.

A user can log in as an administrator or as a regular user (operator). An administrator can choose the tests and change the addresses of virtual instrumentation and other devices. An operator is only allowed to choose which tests will be performed: those for both boards, just for the main one or just for the top board. The sets of tests are predefined in the database for regular users.

The procedure and the order of execution are presented in the block diagram in Fig. 4. The tests are performed sequentally, and the progress can be monitored by the number of finished tests and by the timer that is counting down the time until the approximate end of the verification process (shown on the testing panel in Fig. 5). This time is determined after testing the first set of boards under the new settings and is used everytime after the "NEXT" button is pressed. On the first run the time is set to 6 minutes - the maximum duration of all tests combined.

If all the tests pass, on the main window of the application appears an adequate indication that both boards have passed the verification (two large green circles). The red circle appears in case that one of the boards failed the testing (it fails on the first test with unfavourable outcome) and yellow with a pop-up window in case there is a system error on the instrumentation or within the application.

At the end of the testing, the results can be seen (as shown in Fig. 6), by pressing the button "VIEW RESULTS" on the testing panel. If there is a need to see the boundaries that fulfill the test conditions for the numerical measurements, they will appear as a hint (while hovering the mouse over the wanted value). The tests that are set as not to be performed are shaded. The results are written in the database.

\section{G. MySQL database}

A MySQL database is used for identification of the boards at the beginning of the testing, and for storing of all the relevant information about the boards, such as serial number, hardware and software version, the name of the operator, manufacturing date, as well as the test results (an example of the database results entries can be seen in Fig. 7). The database is easily accessible through the server connection. The login process assumes that all operators are either already defined or can be inserted by an administrator. In the database, there is also the information which tests will be performed if an operator is logged in (option to choose between tests is disabled).

While writing the results in the database, there are 3 states:

- PASS - boards have passed the testing, results of the chosen tests are written in the database, the comment field is empty;

- FAIL - a board has failed the testing (the testing is interrupted after the first test with unfavorable outcome), results of the tests completed to that point are written in the database and the comment field contains a specification of the fail location and type;

- ERROR - there was a system error, i.e. some part of equipment or application was non-operational, the results to that point are written in the database and the comment field contains a specification of the error location and type.

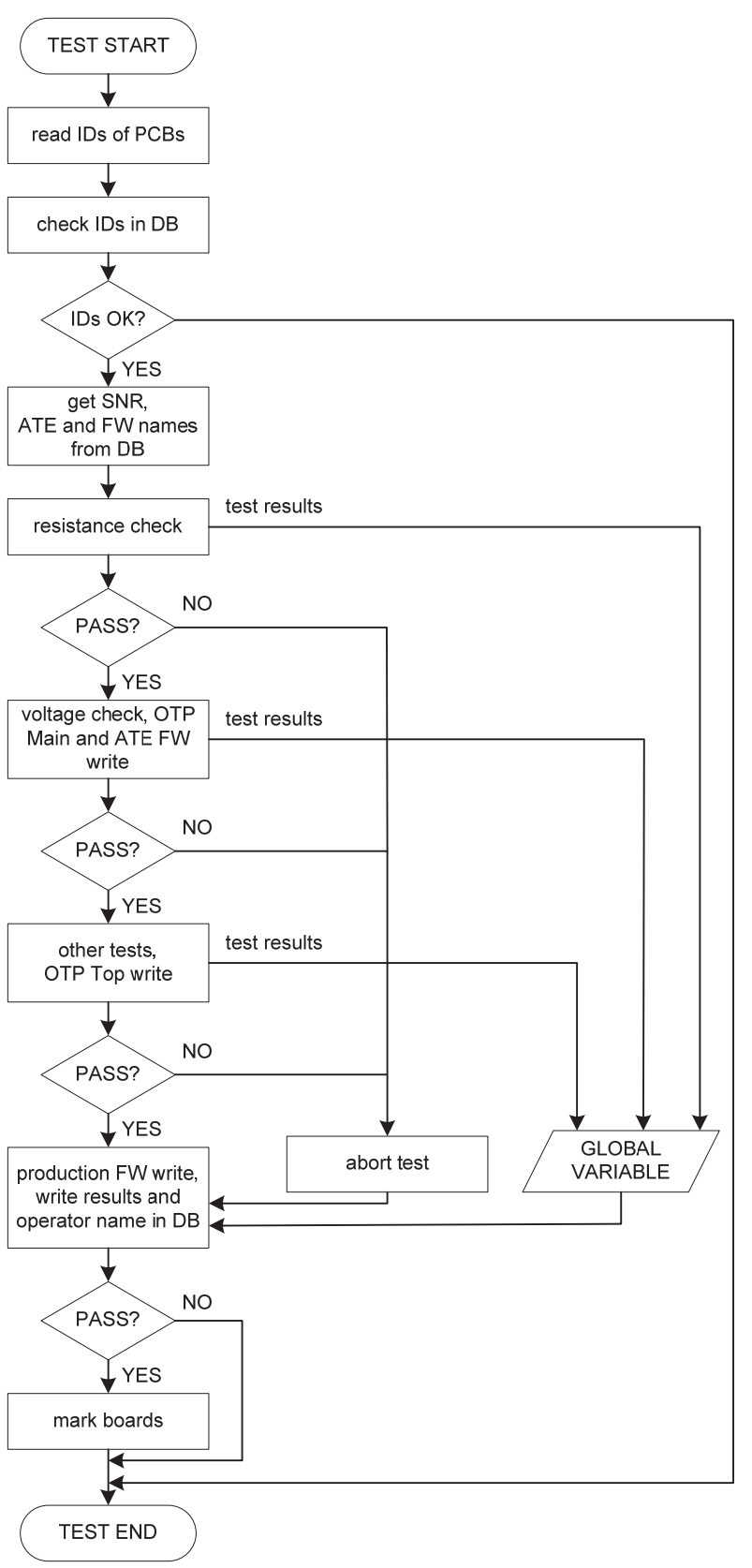

Fig. 4. ATE procedure block diagram. 


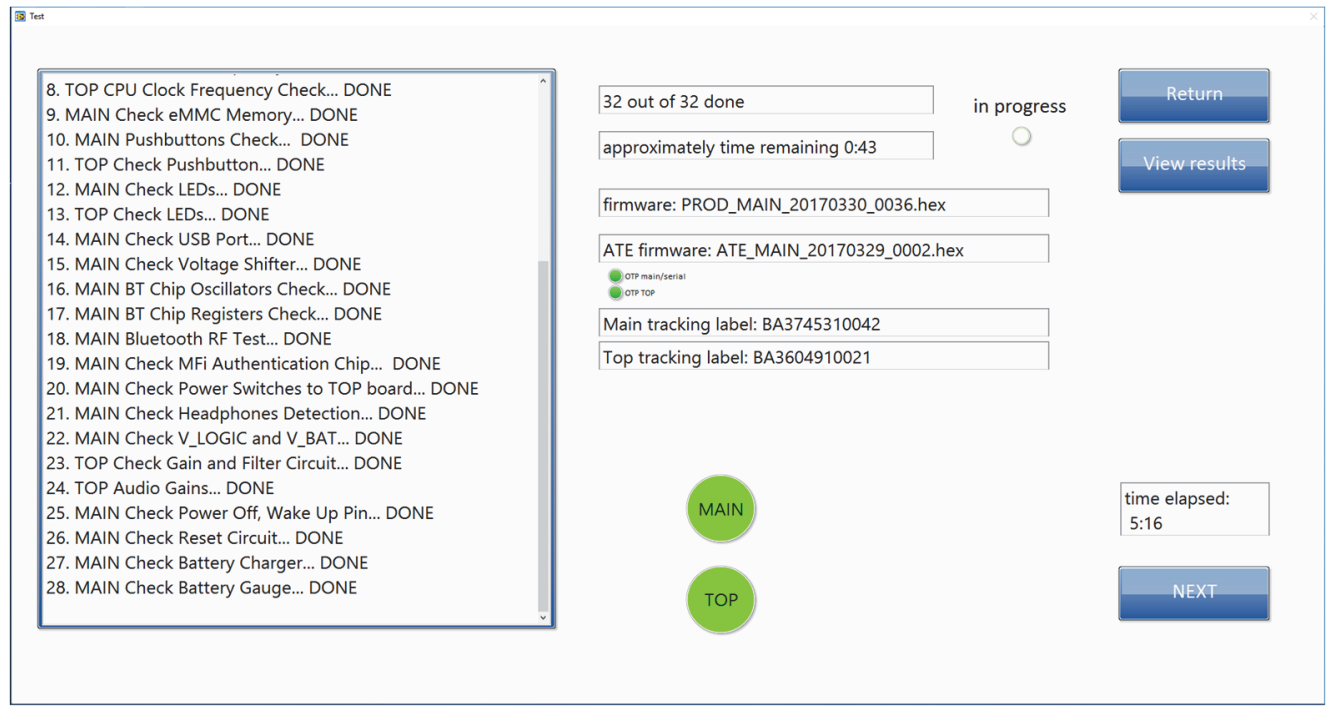

Fig. 5. The testing panel of the application graphical user interface.
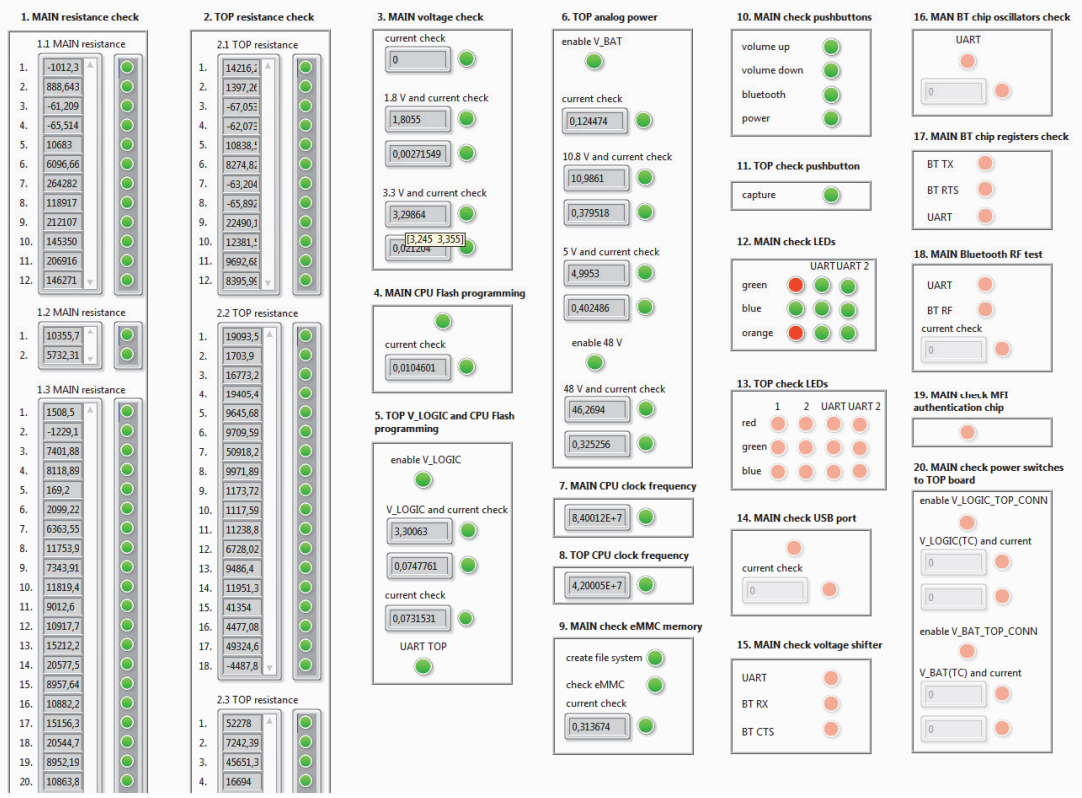

blue 0000
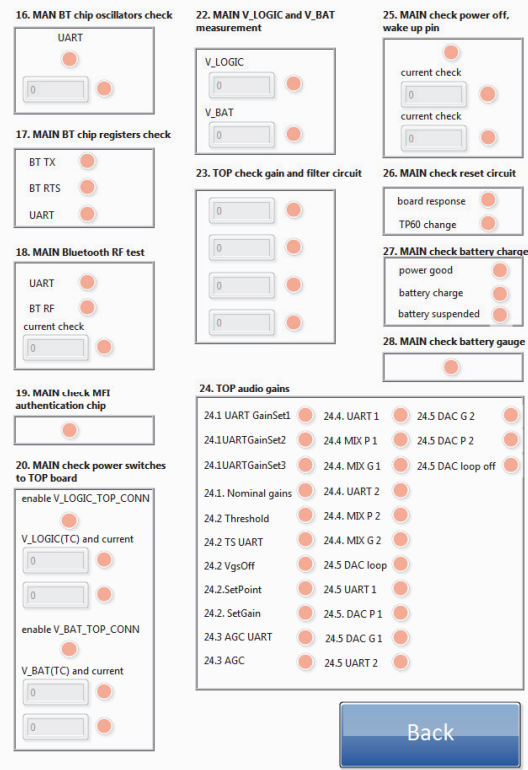

Fig. 6. The results panel of the application graphical user interface (a green indicator - the test passed, a red indicator - the test failed, a shaded indicator - the test not performed).

\begin{tabular}{|c|c|c|c|c|c|c|c|c|c|}
\hline $\begin{array}{l}\text { TESTING TIMESTAMP } \\
\text { Zeststempes de Prefeturg }\end{array}$ & 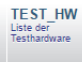 & 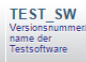 & $\begin{array}{l}\text { TRACKING_LABEL } \\
\text { BA Etikett }\end{array}$ & 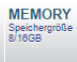 & 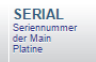 & 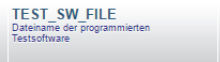 & 001_1_M_R_J3_2_J2_3 & 002_1_M_R_J2_3_J3_2 & 003_1_M_R_J3_1_TP23 \\
\hline 2016-09-16 17:05:04 & 42 & 2.3 & BA3564310847 & $16 \mathrm{~GB}$ & 2601642024 & ATE_MAIN_20160906.0001.hex & $\begin{array}{l}-929,926[-4500,000 / \\
-500,000]\end{array}$ & $\begin{array}{l}839,449[500,000 / \\
4500,000]\end{array}$ & $-32,983[-80,000 /-20,000]$ \\
\hline 2016-09-19 11:14:48 & 42 & 2.3 & BA3564310908 & $16 \mathrm{~GB}$ & 2631642002 & ATE_MAIN_20160906.0001. hex & $\begin{array}{l}-951,320[-4500,000 / \\
-500,000]\end{array}$ & $\begin{array}{l}841,584[500,000 / \\
4500,000]\end{array}$ & $-58,436[-80,000 /-20,000]$ \\
\hline 2016-09-19 11:30:03 & 42 & 2.3 & BA3564310907 & 16GB & 2631642003 & ATE_MAIN_20160906.0001. hex & $\begin{array}{l}-1012,319[-4500,000 / \\
-500,000]\end{array}$ & $\begin{array}{l}888,643[500,000 / \\
4500,000]\end{array}$ & $-61,209[-80,000 /-20,000]$ \\
\hline 2016-09-19 11:50:40 & 42 & 2.3 & BA3564310904 & 16GB & 2631642004 & ATE_MAIN_20160906.0001. hex & $\begin{array}{l}-973,219[-4500,000 / \\
-500,000]\end{array}$ & $\begin{array}{l}852,518[500,000 / \\
4500,000]\end{array}$ & $-55,440[-80,000 /-20,000]$ \\
\hline 2016-09-19 12:02:11 & 42 & 2.3 & BA3564310859 & $16 \mathrm{~GB}$ & 2631642005 & ATE_MAIN_20160906.0001.hex & $\begin{array}{l}-937,384[-4500,000 / \\
-500,000]\end{array}$ & $\begin{array}{l}820,318[500,000 / \\
4500,000]\end{array}$ & $-57,067[-80,000 /-20,000]$ \\
\hline 2016-09-19 12:11:21 & 42 & 2.3 & BA3564310862 & $16 \mathrm{~GB}$ & 2631642006 & ATE_MAIN_20160906.0001. hex & $\begin{array}{l}-971,084[-4500,000 / \\
-500,000]\end{array}$ & $\begin{array}{l}852,760[500,000 / \\
4500,000]\end{array}$ & $-55,361[-80,000 /-20,000]$ \\
\hline 2016-09-19 13:01:25 & 42 & 2.3 & BA3564310918 & $16 \mathrm{~GB}$ & 2631642007 & ATE_MAIN_20160906.0001.hex & $\begin{array}{l}-1003,424[-4500,000 / \\
-500,000]\end{array}$ & $\begin{array}{l}889,150[500,000 / \\
4500,000]\end{array}$ & $-58,015[-80,000 /-20,000]$ \\
\hline 2016-09-19 13:12:52 & 42 & 2.3 & BA3564310914 & 16GB & 2631642008 & ATE_MAIN_20160906.0001.hex & $\begin{array}{l}-979,243[-4500,000 / \\
-500,000]\end{array}$ & $\begin{array}{l}858.973[500,000 / \\
4500,000]\end{array}$ & $-51,280[-80,000 /-20,000]$ \\
\hline 2016-09-19 13:24:02 & 42 & 2.3 & BA3564310919 & 16GB & 2631642009 & ATE_MAIN_20160906.0001.hex & $\begin{array}{l}-952,899[-4500,000 / \\
-500,000]\end{array}$ & $\begin{array}{l}842,374[500,000 / \\
4500,000]\end{array}$ & $-53,473[-80,000 /-20,000]$ \\
\hline 2016-09-19 13:43:56 & 42 & 2.3 & BA3564310906 & $16 \mathrm{~GB}$ & 2631642010 & ATE_MAIN_20160906.0001 hex & $\begin{array}{l}-907,964[-4500,000 / \\
-500,000]\end{array}$ & $\begin{array}{l}793,482[5000,000 / \\
4500,000]\end{array}$ & $-53,109[-80,000 /-20,000]$ \\
\hline 2016-09-19 14:51:00 & 42 & 2.3 & BA3564310915 & $16 G B$ & 2631642011 & ATE_MAIN_20160906.0001.hex & $\begin{array}{l}-921,500[-4500,000 / \\
-500,000]\end{array}$ & $\begin{array}{l}813,523[500,000 / \\
4500,000]\end{array}$ & $-51,740[-80,000 /-20,000]$ \\
\hline 2016-09-19 15:03:07 & 42 & 23 & BA3564310912 & $16 G B$ & 2631642012 & ATE_MAIN_20160906.0001. hex & $\begin{array}{l}-935,152[-4500,000 / \\
-500,000]\end{array}$ & $\begin{array}{l}830,4399[500,000 / \\
4500,000]\end{array}$ & $-51,130[-80,000 /-20,000]$ \\
\hline 2016-09-19 15:21:22 & 42 & 2.3 & BA3564310916 & $16 \mathrm{~GB}$ & 2631642013 & ATE_MAIN_20160906.0001. hex & $\begin{array}{l}-919,681[-4500,000 / \\
-500,000]\end{array}$ & $\begin{array}{l}812,439[500,000 / \\
4500,000]\end{array}$ & $-44,720[-80,000 /-20,000]$ \\
\hline $2016-09-19$ 15:56:28 & 42 & 2.3 & BA3564310905 & 16GB & 2631642014 & ATE_MAIN_20160906.0001. hex & $\begin{array}{l}-964,879[-4500,000 / \\
-500,000]\end{array}$ & $\begin{array}{l}855,924[500,000 / \\
4500,000]\end{array}$ & $-49,755[-80,000 /-20,000]$ \\
\hline 2016-09-19 16:06:13 & 42 & 2.3 & BA3564310857 & 16GB & 2631642015 & ATE_MAIN_20160906.0001. hex & $\begin{array}{l}-998,180[-4500,000 / \\
-500,000]\end{array}$ & $\begin{array}{l}889,064[500,000 / \\
4500,000]\end{array}$ & $-49,120[-80,000 /-20,000]$ \\
\hline 2016-09-19 16:15:37 & 42 & 2.3 & BA3564310913 & $16 G B$ & 2631642016 & ATE_MAIN_20160906.0001.hex & $\begin{array}{l}-944,787[-4500,000 / \\
-500,000]\end{array}$ & $\begin{array}{l}832,928[5000,000 / \\
4500,000]\end{array}$ & $-54,044[-80,000 /-20,000]$ \\
\hline 2016-09-19 16:25:39 & 42 & 2.3 & BA3564310917 & $16 \mathrm{~GB}$ & 2631642017 & ATE_MAIN_20160906.0001. hex & $\begin{array}{l}-944,905[-4500,000 / \\
-500,000]\end{array}$ & $\begin{array}{l}836,512[500,000 / \\
4500,000]\end{array}$ & $-48,615[-80,000 /-20,000]$ \\
\hline 2016-09-19 16:43:40 & 42 & 2.3 & BA3564310861 & $16 \mathrm{~GB}$ & 2631642018 & ATE_MAIN_20160906.0001.hex & $\begin{array}{l}-932,230[-4500,000 / \\
-500,000]\end{array}$ & $\begin{array}{l}827,543[500,000 / \\
4500,000]\end{array}$ & $-52,651[-80,000 /-20,000]$ \\
\hline
\end{tabular}

Fig. 7. An example of the database results entries (a few of main board resistance measurements). 


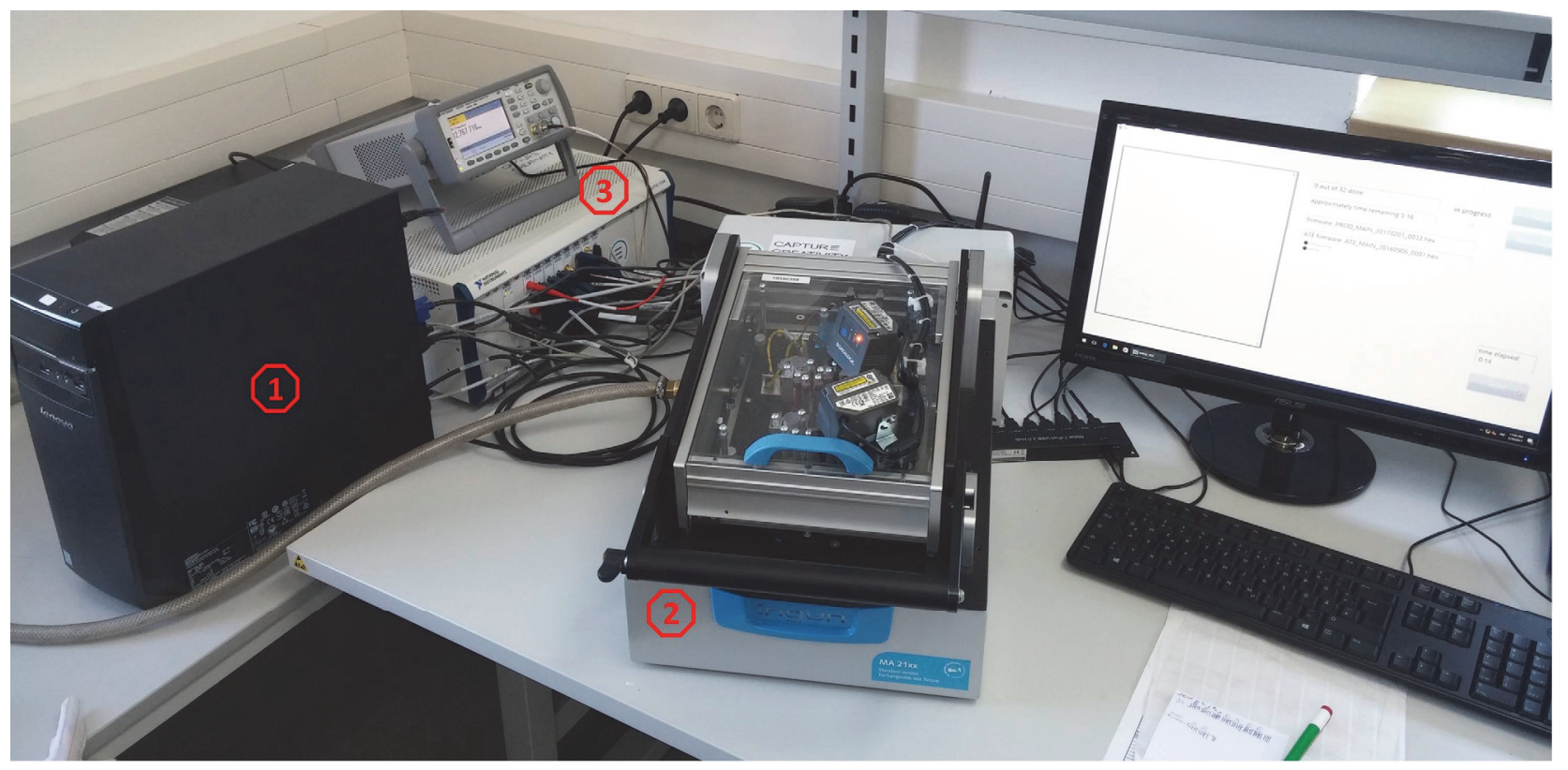

Fig. 8. The automated test environment: 1) a computer with the LabVIEW application, 2) BoN with UUT, 3) NI PXI instrumentation.

\section{TESTING ENVIRONMENT FOR THE MIKME HARDWARE}

Designed test system is used for automatic unit testing in the series production of the MIKME device. Fig. 8 shows the layout of the test environment with units under test. Tests are performed sequentially, checking: resistances, voltages, frequencies, memory, pushbuttons, LEDs, Bluetooth, audio parameters, power supply. As it can be seen, most of the tests comprise some of the basic measurements. However there are more complex tests, such as analysis of the device dynamic range, which requires audio gain measurements. These are necessary due to distinct cut-off voltage between different JFETs causing different gains among devices, and thus are performed in order to provide the same headphones output on every device under the same conditions.

All of the measurement results have to be inside the determined limits. These limits are subject to changes in different production series.

\section{CONCLUSION}

The described automated test system, which is based on a bed-of-nails, ATE firmware, PXI instrumentation, and a LabVIEW application, is successfully applied for units testing in a serial production of the MIKME device. The MIKME device represents a challenge for the testing due to its complexity regarding the verification of the high dynamic range analog channel. The size of the device is a challenge of its own, and it would be quite difficult to test it without a tool of this type.

An average duration of the complete test for both boards is about 6 minutes. From the experience, this time is approximately 20 times shorter in comparison to the time needed for a manual verification and it is also 40 times cheaper.

Until the moment of writing this paper, there were about
2000 main and top boards tested, each. The testing has revealed some irregularities in the production process of the boards.

The final result is that the usage of this system reduces complexity, improves quality and enhances reliability by eliminating the factor of human error.

\section{REFERENCES}

[1] Designing Automated Test Systems - A Practical Guide to SoftwareDefined Test Engineering, National Instruments, 2009.

[2] H. A. Toku, "Developing new Automatic Test Equipments (ATE) using systematic design approaches," 2013 IEEE AUTOTESTCON, Schaumburg, IL, pp. 1-7, 2013.

[3] Automatic Test Equipment on a Budget, Tutorial 761, Maxim Integrated, 2001

Available: https://www.maximintegrated.com/en/ app-notes/index.mvp/id/761

[4] M. Radulović, "Design of an automated test system for a device in a production line," (in Serbian) Master thesis, School of Electrical Engineering, University of Belgrade, 2009.

[5] M. Nešković, J. Spasić, V. Čelebić, I. Salom, "An automated hardware testing using PXI hardware and LabVIEW software," $5^{\text {th }}$ European Conference on Circuits and Systems for Communications ECCSC, Conf. Proc. pp. 232-235, Belgrade, Serbia, November 23-25, 2010.

[6] MIKME GmbH, http://www.mikme.com

[7] G. Ferenc, N. Nenadić, M. Pavlović, S. Trailović, V. Ristić, "MIKME - wireless microphone," Technical Report, Institute Mihailo Pupin, 2015.

[8] National Instruments Corporation, http://www.ni.com

[9] LabVIEW Professional Development System, ver. 2012, National Instruments Corporation

[10] Design guide: Introduction to Automated Test Fixtures, Circuit Check Inc.

[11] INGUN Test Fixtures Catalog 2013/2014. Available: http://ingun.com

[12] V. D. Ćatić, N. M. Lukić, I. M. Salom, V. P. Ristić, M. M. Kabović and N. M. Nenadić, "An automated environment for hardware testing using PXI instrumentation and LabVIEW software," 2016 24th Telecommunications Forum (TELFOR), Belgrade, 2016, pp. 1-4. 